\title{
Ambientes Colaborativos Virtuais: potencial das redes sociais. $O$ caso das empresas do Algarve
}

\author{
Ana Belo ${ }^{1}$, Guilherme Castela ${ }^{2}$, Sílvia Fernandes 3 \\ anagagobelo@gmail.com, gcastela@ualg.pt, sfernan@ualg.pt \\ ${ }^{1}$ SandoGago, Lda. Quarteira, Portugal \\ 2,3 Research Centre for Spatial and Organizational Dynamics, Faculty of Economics - University of Algarve, \\ Campus de Gambelas, 8005-139 Faro, Portugal
}

DOI: 10.4304/risti.12.65-79

\begin{abstract}
Resumo: O objetivo do presente artigo é analisar o potencial das redes sociais no desempenho das pequenas e médias empresas da região do Algarve, tendo sido efectuado um questionário para o efeito. O estudo empírico realizado revela que os dados recolhidos (de 70 empresas) possuem boas qualidades psico-métricas. Procedeu-se a uma análise categórica de componentes principais, a qual identificou duas principais tipologias de objectivos nas redes sociais: redes sociais para interacção produto-cliente e pesquisa ou conhecimento; e redes sociais com potencial para o marketing. Uma análise suplementar - análise hierárquica de clusters (com recurso ao método de agrupamento de Ward) - identificou três padrões de empresas consoante o seu grau de envolvimento em redes sociais: cluster Social Tec Grau 1; cluster Social Tec Grau 2 e cluster Social Tec Grau 3. Estas análises permitem validar uma metodologia sustentável para este tipo de avaliação.
\end{abstract}

Palavras-chave: redes sociais; Internet; padrões; desempenho.

Collaborative Virtual Environments: potential of social networks. The case of companies in the Algarve

\begin{abstract}
The aim of present work is to analyse the potential of social networks in the performance of small and medium enterprises in Algarve region, having developed a questionnaire for this purpose. The empirical study reveals that the collected data (from 70 enterprises) have good psico-metric qualities. Then, a categorical principal component analysis was performed, which identified two main typologies of goals in using social networks: those for product-client interaction and search or knowledge; and those with potential for marketing. A supplementary analysis, hierarchical cluster analysis (using Ward's grouping method), identified three patterns of enterprises according to their level of social networks' involvement: cluster Social Tec Level 1; cluster Social Tec Level 2 and cluster Social Tec Level 3. These analyses validate a sustainable methodology for this kind of approach.
\end{abstract}


Keywords: social networks; Internet; patterns; performance.

\section{Introdução}

A evolução da Internet, em especial o surgimento da web 2.0 (O'Reilly, 2005), abriu novas oportunidades e benefícios para as empresas e para a população em geral, dada a facilidade de comunicação e a velocidade de difusão da informação resultantes (Brandão \& Marques, 2010). Uma das maiores oportunidades foi a abertura das novas aplicações online de ambientes de rede (Tredinnick, 2006; Boyd \& Elisson, 2007; Constantinides et al., 2008). A Internet apresenta-se hoje como a plataforma de maior acesso, à qual actualmente milhões de indivíduos, em qualquer lugar ou momento, acedem diariamente (Tapscott \& Williams, 2007). Neste contexto, em constante mutação, surgem todos os dias novos ambientes e funcionalidades (Evans, 2009) tais como as redes sociais - Facebook, Youtube, LinkedIn, Twitter, Hi5, Bebo, MySpace em que cada vez mais utilizadores comunicam e partilham conteúdos (Pei et al., 2011; Boyd \& Ellison, 2007).

Actualmente muitos indivíduos em Portugal, e no resto do mundo, passam algum tempo do seu dia nestes aplicativos, inclusive durante o horário de trabalho. Nestas plataformas, os indivíduos criam os seus perfis, comunicam, trocam fotos, filmes e conteúdos ou agrupam-se sobre um determinado interesse, criando comunidades. Participar nestas comunidades, influenciando-as em seu benefício ou comunicando com os seus clientes, é de todo o interesse para uma empresa. Tais indivíduos, ligados em rede, participam activamente na inovação, criação de riqueza e desenvolvimento social de uma forma nunca antes pensada (Qualman, 2009).

Segundo o estudo "A utilização da Internet em Portugal 2010" (Taborda, 2010), cerca de $64,8 \%$ dos utilizadores de redes sociais em Portugal consideram importante que as empresas tenham um perfil nessas redes. A entrada das empresas nestes novos aplicativos muda completamente as "regras do jogo". Muitos já sugerem que à Economia do Conhecimento e à Economia Digital, sucede agora uma nova economia com o nome de Socialnomics (Qualman, 2009) ou "Economia das Relações" (Robison \& Ritchie, 2010), ou ainda "Economia da Integridade" (Bernasek, 2010). Essa nova Economia baseia-se em inter-relacionamentos tendo como alicerce a confiança e a integridade. Os recursos fundamentais na empresa, que em séculos passados eram tangíveis, são agora substituídos por valores intangíveis como o conhecimento, a confiança e as relações entre indivíduos. As empresas tentam adaptar-se a estas revoluções e inovações de forma a retirar valor acrescentado.

O despertar das empresas e organizações para estas ferramentas sociais é uma reacção recente. Os estudos das redes sociais têm sido ampliados, recebendo cada vez mais atenção da comunidade científica (Boyd \& Ellison, 2007). Estes sítios web constituem presentemente um importante foco de pesquisa para trabalhos de investigação em diversas áreas. Um dos exemplos que se pode dar é o aplicativo Facebook, que já foi estudado por Dwyer et al. (2007); Acquisti \& Gross (2006); Lampe et al. (2007) e Stutzman (2006).

Com este propósito, é fundamental o estudo das inter-relações entre empresa e redes sociais no âmbito do seu potencial, especialmente para as pequenas e médias empresas 
(PME). Nesse sentido, interessa caracterizar actualmente a sua participação nestes sítios web inovadores, bem como analisar que actividades ficam mais potenciadas. Algumas empresas da região do Algarve utilizam estas redes na actividade de marketing. Mas as empresas não devem ver as redes sociais apenas como uma moda ou uma estratégia de marketing online (Evans, 2009). Precisam olhar mais fundo e analisar estes novos ambientes com uma perspectiva inovadora, pois as redes sociais constituem um meio de comunicação que abrange milhões de potenciais clientes (Vasconcelos \& Campos, 2010; Tapscott \& Williams, 2007; Brandão \& Marques, 2010; Constantinides et al., 2008). A adaptação das empresas a estas novas realidades implica uma inovação na sua estratégia e organização (até no design organizacional).

\section{Estudos Comparados}

As redes sociais introduziram mudanças fundamentais na forma de comportamento dos utilizadores. Algumas empresas mais inovadoras reconheceram essa mudança e estão a tirar partido de serem as primeiras a divulgar e promover as suas actividades nas redes sociais participando, criando comunidades e comercializando seus produtos online (Evans, 2009). Destas mudanças advêm grandes vantagens para as empresas, dado que a tecnologia é uma das principais ferramentas utilizadas pelas empresas para inovar. Diversos estudos empíricos comprovam as ligações entre TI, inovação e o sucesso competitivo das PME (Edquist \& Henrekson, 2006).

Estes ambientes têm a vantagem de permitir uma comunicação a baixo custo, o que conduz a uma enorme adesão, fazendo com que as redes rapidamente se expandam conectando utilizadores em todo o mundo (Hempel, 2009; Golder et al., 2007). O que mais leva os indivíduos a agruparem-se nestas ferramentas sociais é o facto de partilharem interesses em comum (Shirky, 2010; Weber, 2009) sem contacto físico ou sem precisarem encontrar-se no mesmo espaço físico (Kardaras et al., 2003).

Uma previsão efectuada por Wilson (2009) indica que 50\% dos utilizadores de Internet no Reino Unido visitarão as redes sociais pelo menos uma vez por mês até 2013, e trabalhadores de escritório pelo menos 30 minutos por dia (Yap \& Robben, 2010). As PME não podem ignorar o tempo utilizado nas redes sociais e, como tal, devem ir ao encontro dessas comunidades. Não podem ignorar que estas representam benefícios no lançamento de produtos inovadores, entre outras oportunidades como: promoções (Evans, 2009); pesquisa e suporte técnico (Wilson, 2009); confiança, cooperação, lançamento de concursos, fidelização, pedidos de opinião, novos negócios, informação relevante para o negócio, recrutamento (Pei et al., 2011); internacionalização (Zhou et al., 2007) e criação/ distribuição informal de sistemas de informação para inovação (Vasconcelos \& Campos, 2010). E ainda Arima (2010) valoriza os media sociais como uma oportunidade para as organizações construírem marcas, demonstrarem comportamentos de liderança, expandirem recursos, alcançarem novas audiências e encontrarem novas fontes de ideias.

O estudo de Ingelbrecht et al. (2010), utilizando uma amostra de 4.000 consumidores em 10 mercados mundiais (incluindo Estados Unidos, França, Alemanha e China) remete para as redes sociais como o Facebook e o LinkedIn, a possibilidade de se afirmarem como ferramentas importantes de retalho e compras. O mesmo estudo indica que as empresas podem utilizar a colaboração em massa como elo entre o valor 
de negócio e estas tecnologias. Por exemplo, podem examinar uma comunidade de destino de determinado produto e interagir de modo a formular novas maneiras de vender o produto, ou de inovar esse produto, acrescentando valor para a empresa. Ou seja, aproveitar a "inteligência colectiva" da comunidade para alcançar eficácia operacional (Levy, 1990).

Um estudo comentado por Falcão (2010), da IGMarketing, conclui que as redes sociais são um conjunto de ferramentas que beneficiam a empresa numa relação proporcional ao seu investimento. Assim, é necessário saber como participar, investir tempo, criar conteúdos, incrementar a rede. Daí resultam competências para a equipa e para a actividade individual dos trabalhadores. Algumas empresas estão mesmo a eliminar as barreiras entre o online e o offline, contratando profissionais nestas áreas (E.life, 2010), de modo a migrá-las para as redes sociais, ficando o seu sítio web (site) numa linha estratégica secundária.

$\mathrm{O}$ aspecto mais diferenciador desta inter-relação empresa e redes sociais é o compromisso (engagement) com a comunidade (Cross \& Thomas, 2010). Os benefícios dessa representação ou participação nas redes sociais só existem se as empresas apreenderem o poder do comportamento colectivo no seu desempenho (Bradley, 2011). É importante encontrar o "momento social”, ou seja, a dinâmica social que, utilizando as especificidades da Internet e de sua interactividade, potencia o valor económico do modelo de negócio (Hummel \& Lechner, 2002).

\section{Estudo Empírico}

\subsection{Introdução e Metodologia Aplicada}

Começou-se por uma observação do mundo real, através de um estudo prático dos sítios web de redes sociais e da representação de empresas nestes, bem como uma análise de como as empresas estão sendo beneficiadas com tais relações. Nesse sentido, decorreu uma fase de comparação de diversos vídeos, apresentações disponíveis e grupos de discussão sobre o tema, seguindo-se uma fase de análise de estudos empíricos já efectuados. Na concepção do plano de investigação reflectiu-se em alguns objectivos que seriam mais pertinentes para as empresas. Seguiu-se uma selecção das técnicas de recolha de informação e de análise de dados. Na recolha de informação, optou-se por fazer uma recolha de dados com recurso ao preenchimento de um questionário, visando obter resultados relevantes, após a análise dos dados obtidos.

Os dados recolhidos foram depois tratados por meio de uma análise quantitativa. Como tal, utilizamos a técnica de Análise Categórica de Componentes Principais (CATPCA). Numa primeira fase, a CATPCA foi utilizada para detectar padrões de associação nas variáveis obtidas, transformadas em escalas óptimas (Gifi, 1990; De Leeuw, 1990; Meulman, 1992; Nishisato, 1994). Numa segunda fase, foi utilizada a análise hierárquica de Clusters (Romesburg, 1984) com recurso ao método Ward como critério de agrupamento para detectar padrões de associação nas empresas, tendo em atenção as variáveis realçadas pela análise anterior (CATPCA). Existem outros estudos que já conjugaram estes dois métodos de análise, como por exemplo o de Sousa et al. (2003). 


\subsection{Amostra e Questionário}

Tendo em mente a validação da recolha de dados, foi dirigido um questionário às PME do Algarve (QPME). Este tem como objectivo caracterizar as tipologias de PME da região do Algarve na sua relação com os ambientes de redes sociais. Utilizou-se para tal uma ferramenta especializada - Surveymonkey (www.surveymonkey.com) - que permite conceber um sítio web onde o questionário fica disponível. $\mathrm{O}$ uso desta ferramenta online na investigação permite um acesso rápido aos questionários, facilitando uma maior rapidez de resposta.

Os dados foram recolhidos entre Outubro e Dezembro de 2010. Era dada aos participantes a oportunidade de reverem as respostas. Um e-mail de apresentação, com a introdução do link para o referido questionário, foi enviado às PME do Algarve. Alguns dos endereços de e-mail foram retirados das páginas amarelas, sendo um critério utilizado verificar se a empresa era mesmo do Algarve. As respostas são dadas directamente no Surveymonkey e os dados obtidos são confidenciais, podendo apenas ser acedidos com username e password, sendo encriptados entre servidor e cliente (Minayo et al., 2007).

Foram obtidos 70 casos de empresas algarvias, respondidos no prazo estipulado. Algumas empresas foram contactadas por meio de quatro instituições que mantêm contactos com as PME da região (CRIA, NERA, CIEO e Directório Empresarial Sulempresas.com). Depois de fechado o processo dos questionários online, recolheramse os dados para serem tratados. Tal tratamento começou por ser feito pela própria ferramenta Surveymonkey, sendo complementado numa discussão posterior pelas análises estatísticas desenvolvidas no SPSS v.17. As variáveis usadas nessas análises correspondem às várias questões do QPME.

\section{Análise de Padrões}

O objectivo desta análise é chegar a um diagnóstico das variáveis mais relevantes na participação das empresas em estudo nas redes sociais. Para isso, utilizou-se a CATPCA - Análise Categórica de Componentes Principais (Gifi, 1990; De Leeuw, 1990; Meulman, 1992; Nishisato, 1994) como técnica exploratória de interdependência e de redução da dimensionalidade dos dados. O objectivo é detectar padrões de associação nas variáveis. Esta análise, aplicada aos dados recolhidos, permitiu obter resultados bastante consistentes conforme mostra a Tabela 1:

Tabela 1 - Sumário do modelo

\begin{tabular}{lll}
\hline Dimensãoo & $\begin{array}{l}\text { Alphade } \\
\text { Cronbach }\end{array}$ & $\begin{array}{l}\text { Variância obtida } \\
\text { (Eigenvalue) }\end{array}$ \\
\hline 1 & 0,899 & 8,353 \\
\hline 2 & 0,774 & 4,139 \\
\hline Total & 0,940 & 12,492 \\
\hline
\end{tabular}

De acordo com o coeficiente Alpha de Cronbach, indicador do grau de consistência interna das observações, verifica-se que a amostra revela uma elevada consistência 
interna em duas dimensões (num total de 94\%). A literatura relacionada indica que a consistência interna é significativa se estiver acima de 70\% (Gifi, 1990; De Leeuw, 1990; Meulman, 1992; Nishisato, 1994). Ainda se observa a variância explicada, que na $1^{\mathrm{a}}$ dimensão é de $66,86 \%$ e na $2^{\mathrm{a}}$ dimensão de $33,13 \%$ (estes valores obtêm-se dividindo a variância obtida pela variância total).

A CATPCA revelou ainda as ponderações que cada uma das variáveis apresenta. A Figura 1 ilustra a estrutura bidimensional de distribuição das variáveis no espaço de referência, na qual os vectores projectam as cargas das variáveis.

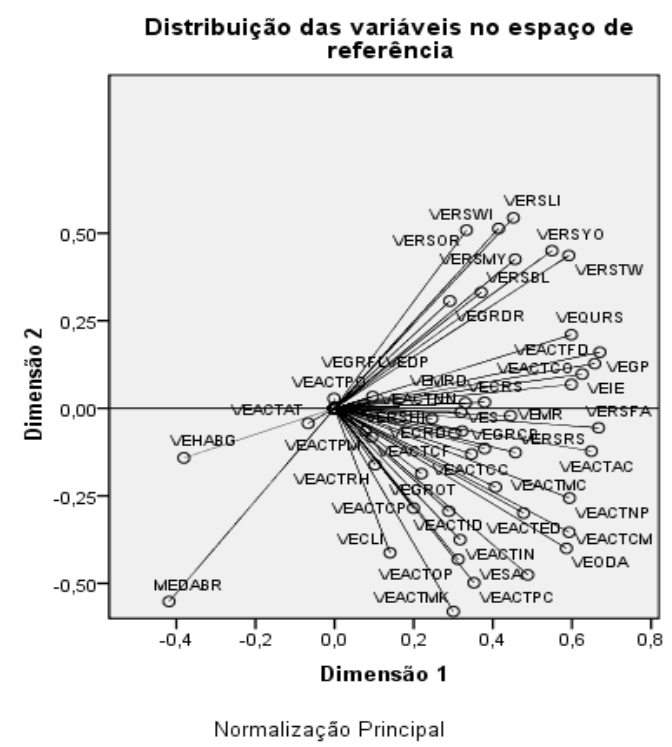

Figura 1 - Distribuição Bidimensional das Variáveis

Por outro lado, quanto aos casos observados (empresas), a Figura 2 ilustra a estrutura bidimensional de distribuição das empresas no mesmo espaço de referência: 


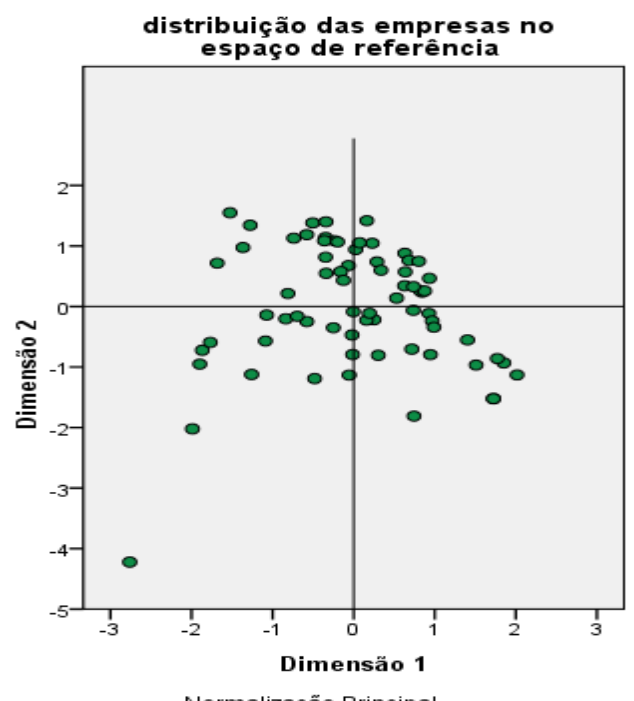

Figura 2 - Distribuição Bidimensional das Empresas

No que diz respeito às variáveis, a Tabela 2 descreve os respectivos valores de carga nas duas dimensões (sendo as cargas um reflexo da importância relativa das variáveis). Os autores das referências atrás mencionadas defendem que o critério de relevância da variável ocorre quando a sua carga está acima de 0,5 em pelo menos uma das dimensões. Assim, a Tabela 2 assinala a diferentes cores as cargas das variáveis (mais relevantes) na $1^{\mathrm{a}}$ dimensão e na $2^{\mathrm{a}}$ dimensão respectivamente:

Tabela 2 - Cargas das variáveis relevantes

\begin{tabular}{lll}
\hline Variável & \multicolumn{2}{l}{ Dimensões } \\
\hline VERSOR & 0,333 & 0,51 \\
\hline VERSWI & 0,415 & 0,51 \\
\hline VERSFA & 0,67 & $-0,056$ \\
\hline VERSTW & 0,59 & 0,436 \\
\hline VERSLI & 0,452 & 0,54 \\
\hline VERSYO & 0,55 & 0,45 \\
\hline VERSRS & 0,65 & $-0,123$ \\
\hline VEIE & 0,63 & 0,098 \\
\hline VEODA & 0,59 & $-0,4$ \\
\hline VEACTMK & 0,3 & $-0,58$ \\
\hline
\end{tabular}




\begin{tabular}{lll}
\hline VEACTNP & 0,59 & $-0,256$ \\
\hline VEACTFD & 0,66 & 0,128 \\
\hline VEACTCM & 0,59 & $-0,354$ \\
\hline VEACTCO & 0,6 & 0,068 \\
\hline VEGP & 0,67 & 0,16 \\
\hline VEQURS & 0,6 & 0,21 \\
\hline
\end{tabular}

Nesta tabela, outro aspecto a mencionar é que a maior parte das variáveis tem valores positivos na $1^{\mathrm{a}}$ dimensão, enquanto a $2^{\mathrm{a}}$ dimensão revela um maior contraste entre as variáveis pois umas são negativas e outras positivas, significando que há poucas relações entre elas. Pode-se daí reunir mais fundamentos para as tipologias de redes sociais encontradas e para os padrões de empresas no seu envolvimento com as mesmas. Na Tabela 3 pode observar-se que a avaliação das principais variáveis originou as seguintes tipologias de redes sociais:

Tabela 3 - Tipologias de redes sociais (dimensões de variáveis relevantes)

\begin{tabular}{ll}
\hline Dimensões (tipologias) & Caracterização \\
\hline $\begin{array}{l}\text { Dimensão 1: redes sociais para } \\
\text { interacção produto-cliente }\end{array}$ & $\begin{array}{l}\text { Twitter, Youtube - mais orientadas à pesquisa, } \\
\text { comunicação e conhecimento (mais }\end{array}$ \\
& $\begin{array}{l}\text { profissionais); e } \\
\text { Fesquisa ou conhecimento }\end{array}$ \\
& $\begin{array}{l}\text { Facebook - mais orientada à interacção com } \\
\text { clientes, novos produtos a clientes, desempenho } \\
\text { (mais sociais) }\end{array}$ \\
\hline Dimensão 2: redes sociais com & Orkut, Wikis, LinkedIn - mais orientadas ao \\
potencial para o marketing & marketing e divulgação \\
\hline
\end{tabular}

Tendo por base as Tabelas 2 e 3, verifica-se que a primeira dimensão obtida retém mais informação pois capta a maior percentagem da variância (Tabela 1). A segunda dimensão retém menos informação e capta a variância residual (Gifi, 1990; Romesburg, 1984). O objectivo da análise utilizada é reduzir a dimensionalidade dos dados com a menor perda de informação possível. Ao extrairmos duas dimensões perde-se informação, contudo a utilização de todas as dimensões complicaria a análise. Nesse sentido, a Dimensão 1 (redes sociais para interacção produto-cliente e pesquisa ou conhecimento) está bem caracterizada na Tabela 3, tendo em conta as variáveis cujos valores de cargas são todos positivos (Tabela 2). No entanto a Dimensão 2, dado que o valor de carga da variável relacionada com o marketing (VEACTMK) é negativo, designamo-la como redes sociais com “potencial” para marketing. Também as relações entre as variáveis desta dimensão são fracas, como atrás referido.

Especialistas em e-business e social media defendem tais dimensões mas sublinham que não há trabalhos empíricos em Portugal que sustentem claramente a sua ligação com os tipos de redes sociais e objectivos descritos (Tabela 3). Existem no entanto grupos de discussão, como por exemplo os grupos "Social network/media strategy" e "Social media Portugal" do LinkedIn, onde estes aspectos são debatidos. Contudo, 
limitam-se a opiniões de especialistas que habitualmente trabalham nestas áreas, revelando considerável experiência mas sem suporte empírico sustentável.

Segue-se, através da Figura 3, a apresentação da estrutura conjunta de distribuição das variáveis e empresas, no espaço de referência considerado de relevância. Nesta estrutura bidimensional podem ser detectados três clusters de PME através de uma análise hierárquica de clusters com recurso ao método de agrupamento Ward (Romesburg, 1984). Para que a Figura 3 não fique confusa, aparece nela apenas o Cluster 1 estando os restantes Clusters representados na Figura 4.

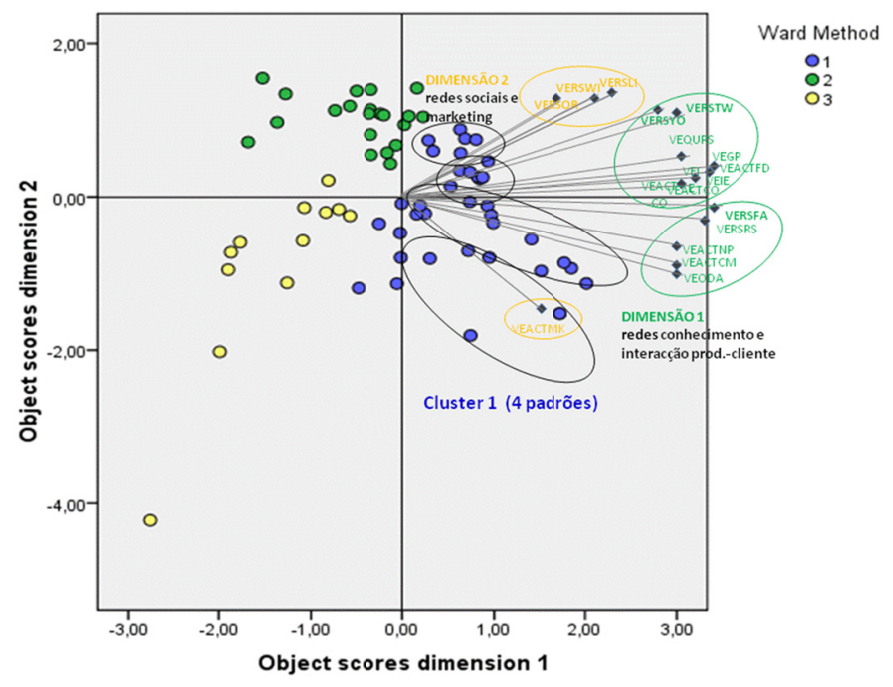

Figura 3 - Distribuição Bidimensional das Variáveis e Empresas (dimensões/clusters) 


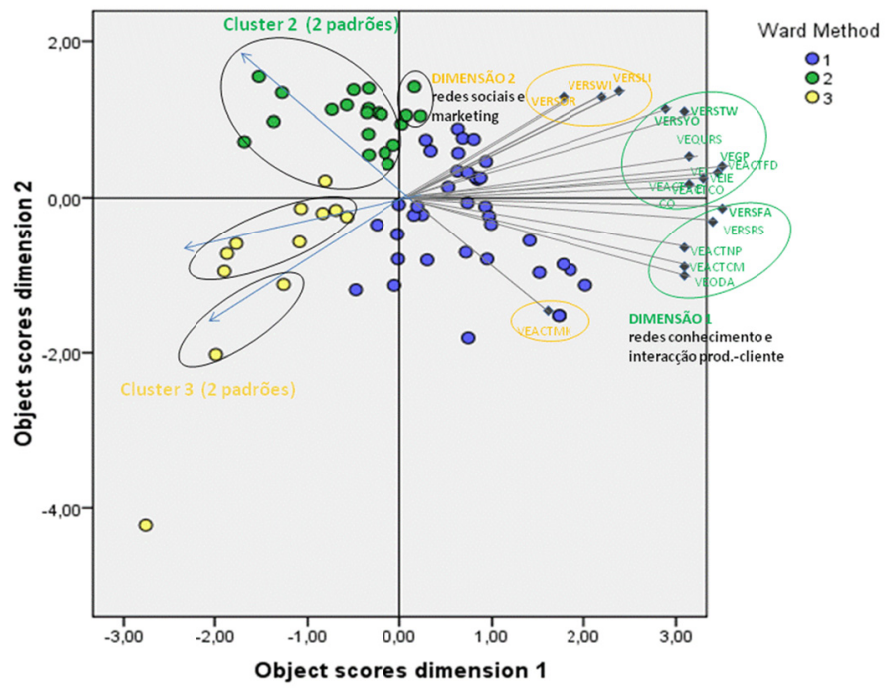

Figura 4 - Distribuição Bidimensional das Variáveis e Empresas (dimensões/clusters) Cont.

Comparando as Figuras 3 e 4, os três clusters de PME obtidos correspondem a padrões de comportamento das mesmas consoante as variáveis referentes às actividades por elas praticadas nas redes sociais. É de notar que os clusters estão por sua vez divididos em sub-padrões (o cluster 1 em 4 sub-padrões; e os clusters 2 e 3 em 2 sub-padrões respectivamente). As características específicas de tais sub-padrões serão alvo de futura investigação. Com base nas variáveis relevantes, e na importância estatística dada pela CATPCA, os três clusters encontrados foram denominados segundo o grau de envolvimento das empresas nas redes sociais:

CLUSTER SOCIAL TEC GRAU 1 - formado por 37 empresas, que maioritariamente verificam oito características:

- Ausência de presença nas redes sociais Wiki, Facebook, Twitter, LinkedIn, Youtube (ausência total de presença no Orkut);

- Habitualmente não recorrem a redes sociais no melhor desempenho de suas actividades;

- Não implementaram uma estratégia integrada com redes sociais;

- Existência de uma opinião favorável ao desempenho da empresa pela utilização das redes sociais;

- Existe uma fraca actividade no que se refere ao marketing, lançamento de novos produtos, fidelização e análise de padrões de comportamento;

- Existe uma forte actividade no que se refere à comunicação com os clientes;

- Ausência de um grupo de indivíduos na empresa envolvidos na representação desta nas redes sociais; 
- Quando recorrem às redes sociais, fazem-no apenas 1 vez por semana.

CLUSTER SOCIAL TEC GRAU 2 - formado por 21 empresas, que maioritariamente verificam cinco características:

- Uma presença muito significativa no Facebook;

- Habitualmente recorrem a redes sociais no melhor desempenho de suas actividades;

- Existe uma forte actividade no que se refere ao lançamento de novos produtos e comunicação com os clientes;

- Verifica-se ainda uma total prática da actividade de divulgação nas redes sociais, bem como uma opinião generalizada favorável ao desempenho das empresas pela utilização destas redes;

- Recorrem às redes sociais todos os dias na prática de suas actividades.

CLUSTER SOCIAL TEC GRAU 3 - formado por 12 empresas, que maioritariamente verificam seis características:

- Ausência de presença nas redes sociais Orkut e Wiki;

- Uma presença muito significativa no Twitter, LinkedIn, Youtube;

- De modo geral já implementaram estratégias integradas com as redes sociais;

- Existe uma forte actividade no que se refere à fidelização e análise de padrões de comportamento;

- Existência de um grupo de indivíduos envolvidos na representação da empresa nas redes sociais;

- Recurso às redes sociais várias vezes por dia.

É de realçar que, tendo em conta a Figura 3, as empresas do Cluster 1 parecem em geral verificar as características (variáveis) da Dimensão 1 de redes sociais (pois o dito Cluster e variáveis estão do lado direito da estrutura). Contudo, ao analisar as características dos clusters atrás descritas, constata-se que as empresas do Cluster 1 não estão em geral representadas nas redes sociais. Isto porque os quadrantes do lado direito daquela estrutura bidimensional apresentam negatividade nas variáveis ('Não').

\section{Conclusões}

As redes sociais são, actualmente, objecto de muita discussão dada a adesão em massa de indivíduos e empresas. O presente estudo, incidindo nas PME, pretendeu reunir duas abordagens: investigar se as PME do Algarve já utilizam essas redes no melhor desempenho de suas actividades, e analisar as características e potencial dessa actuação. Para tal foi dirigido um questionário às PME da região do Algarve. O estudo empírico elaborado constatou que estas utilizam as redes sociais para diversas actividades. Através de uma Análise Categórica de Componentes Principais, o estudo identificou duas dimensões ou tipologias de redes sociais: redes para interacção 
produto-cliente e pesquisa ou conhecimento; e redes sociais com potencial para marketing. Na primeira dimensão, as redes sociais que mais foram selecionadas pelas PME são: o Facebook, rede mais orientada à interacção com clientes, novos produtos a clientes e desempenho (mais social); e o Twitter e Youtube, redes mais orientadas à pesquisa, comunicação e conhecimento (mais profissionais). Na segunda dimensão, o destaque foi para as redes Orkut, Wikis e LinkedIn, mais orientadas ao marketing e divulgação. No entanto, as associações são fracas nesta segunda dimensão.

Por sua vez, o envolvimento das empresas algarvias nas redes sociais não é uniforme. $\mathrm{Na}$ amostra de PME estudada encontramos três padrões através da Análise Hierárquica de Clusters (com recurso ao método Ward como critério de agrupamento), denominados de: Cluster Social Tec Grau 1; Cluster Social Tec Grau 2; Cluster Social Tec Grau 3. No primeiro cluster as empresas têm menor envolvimento com as redes sociais do que nos outros clusters. No entanto, o caso de uma empresa que se encontre no segundo cluster não significa que está "atrasada" na sua actuação nas redes sociais em relação às do terceiro cluster. Significa sim que existe um padrão de envolvimento diferente, pois os clusters 2 e 3 têm ambos empresas com presenças significativas nas redes sociais. Porém, as empresas do terceiro cluster são as que medem resultados dessa presença e já têm uma estratégia integrada, recorrendo várias vezes ao dia às redes sociais.

Uma das limitações deste trabalho é que a própria literatura nesta área é limitada, não existindo em Portugal trabalhos empíricos que sustentem claramente as dimensões obtidas na sua ligação com as tipologias de redes sociais e objectivos descritos. Existem sim grupos de discussão onde estes aspectos são debatidos por profissionais que habitualmente trabalham nestas áreas. Outra limitação tem a ver com a dimensão da amostra de empresas recolhida. A análise dos resultados obtidos permite indicar algumas recomendações para pesquisas futuras. Uma ideia seria estudar o contributo das plataformas sociais, como o LinkedIn, no recrutamento dos indivíduos. Em áreas transdisciplinares, as redes sociais podem motivar os trabalhadores no sentido de pesquisar novas ideias de melhoria dos seus trabalhos ou propor novos procedimentos nas instituições onde trabalham. Na área da governação (governance), sugere-se um estudo sobre o potencial das redes sociais no sentido de: maior comunicação a nível de cidadãos; fortalecer laços sociais; lutar contra a pobreza; procura de emprego; e criação de sistemas de auxílio mútuo (Frey, 2000; Shirky, 2010). Seria ainda útil um estudo para analisar que políticas podem auxiliar as PME em Portugal a implementar os seus negócios tendo em vista a quantidade de utilizadores presentes nas redes sociais.

\section{Referências}

Acquisti, A. \& Gross, R. (2006). Imagined Communities: Awareness, Information Sharing, and Privacy on the Facebook. Acedido em: 03/02/2011. Disponível em: $<$ http://www.heinz.cmu.edu/ acquisti/papers/acquisti-gross-facebook-privacyPET-final.pdf $>$.

Arima, K. (2010). Dicas sobre o Uso de Redes Sociais na Empresa. Acedido em: 02/04/2011. Disponível em: <http://info.abril.com.br/noticias/corporate/ gartner/dicas-sobre-uso-de-redes-sociais-na-empresa-28062010-13.shl>. 
Bernasek, A. (2010). The Economics of Integrity. Editora Harperstudio.

Boyd, D. \& Ellison, N. (2007). Social Network Sites: Definition, History, and Scholarship. Journal of Computer-Mediated Communication, 13(1), article 11. Acedido em: 20/02/2011. Disponível em: <http://jcmc.indiana.edu/vol13/ issue1/boyd.ellison.html>.

Bradley, A. (2011). Employing social media for business impact: Key collective behavior patterns. Acedido em 12/03/2011. Disponível em: <http://www.gartner.com/DisplayDocument?ref=clientFriendlyUrl\&id=1538316>

Brandão, R. \& Marques, B. (2010). The impact of web 2.0 in the informal learning of the Portuguese SME. AIP-CE, CEPCEP, Universidade Católica Portuguesa.

Constantinides, E., Lorenzo, C. \& Gómes-Borja, M. (2008). Social media: A new frontier for retailers?, European Retail Research, 22, 1-27.

Cross, R. \& Thomas, R. (2010). Conduzir o desempenho através das redes sociais. Editora Vida Económica.

De Leeuw, J. (1990). Multivariate analysis with optimal scaling, in Gupta, S. e Sethuraman, J. (Eds.) Progress in Multivariate Analysis. Calcutta: Indian Statistical Institute.

Dwyer, C., Hiltz, C. \& Passerini, K. (2007). Trust and privacy concern within social networking sites: A comparison of Facebook and MySpace. America's Conference on Information Systems (AMCIS), USA.

Edquist, H. \& Henrekson, M. (2006). Technological breakthroughs and productivity growth. Acedido em: 22/02/2011. Disponível em: <http:// www.ifn.se/Wfiles/wp/wp665.pdf>.

E.life (2010). O Futuro das redes sociais: Fim das barreiras offline. Acedido em: 07/03/2011. Disponível em: <http://www.itweb.com.br/noticias/index.asp? $\operatorname{cod}=74536>$.

Evans, D. (2009). Marketing de media social, uma hora por dia. Editora AltaBooks.

Falcão, H. (2010). Social technology. Acedido em 15/02/2011. Disponível em: $<$ http://pt.scribd.com/collections/2545330/Social-Technology-TecnologiaSocial $>$.

Frey, K.(2000). Governança electrónica: Experiências de cidades europeias e algumas lições para países em desenvolvimento. Acedido em 20/03/2011. Disponível em: <http://www.ip.pbh.gov.br/ANO3_N1_PDF/ipo301frey.pdf > .

Gifi, A. (1990). Nonlinear multivariate analysis. New York: J. Wiley \& Sons.

Golder, S., Wilkinson, D \& Huberman, B. (2007). Rhythms of social interaction: Messaging within a massive on-line network. Proceedings of the 3 rd International Conference on Communities and Technologies. London, UK. 
Hempel, J. (2009) How facebook is taking over our lives. Acedido em 16/03/2011. Disponível em: <http://money.cnn.com/2009/02/16/technology/ hempel_facebook.fortune/index.htm $>$.

Hummel, J. \& Lechner, U. (2002). Social profile of virtual communities. Proceeding of the 35th Hawaii International Conference on Systems Sciences. Hawaii.

Ingelbrecht, N., Patrick, C. \& Foong, K. (2010). User survey analysis: Consumer marketing using social network analysis. Acedido em: 20/03/2011. Disponível em: <http://www.gartner.com/resId=1381514>.

Kardaras, D, Karakostas, B \& Papathanassiou, E. (2003). The potential of virtual communities in the insurance industry in the UK and Greece, International Journal of Information Management, 23(1), 41-53.

Lampe, C., Ellison, N. \& Steinfeld, C. (2007). Profile elements as signals in an on-line social network. Conference on human factors in computing systems. San Jose, CA, USA.

Levy, P. (1990). Les technologies de l’intelligence. Paris: Editora la Découverte.

Meulman, J. (1992) The integration of multidimensional scaling and multivariate analysis with optimal transformations of the variables, Psychometrika, 57, 539565 .

Minayo, M., Suely F. \& Gomes, R. (2007). Pesquisa social, teoria, método e criatividade. Brasil: Petrópolis.

Nishisato, S. (1994). Elements of dual scaling: An introduction to practical data analysis. New Jersey: Lawrence Erlbaum Associates, Inc.

O'Reilly, T. (2005). What is web 2.0: Design patterns and business models for the next generation of software. Acedido em: 09/04/2011. Disponível em: <http://www.elisanet.fi/aariset/Multimedia/Web2.o/What\%20Is\%20Web\%202. doc $>$.

Pei, M., Ramayah, T. \& Suki, N. (2011). Factors enhancing employed job seekers intentions to use social networking sites as a job search tool, International Journal of Technology and Human Interaction, 7(2), 38-54.

Qualman, E. (2009). Socialnomics - Como os media sociais estão a mudar o mundo como vivemos e como fazemos negócios. Editorial Presença.

Robison, L. \& Ritchie, B. (2010). Relationship economics: The social capital paradigm and its application to business, politics and other transactions. Editora Gower.

Romesburg, H. (1984). Cluster analysis for researchers. USA: Lifetime Learning Publications.

Shirky, C. (2010). Eles vêm aí - O poder de organizar sem organizações. Actual Editora.

Sousa, L., Galante, H. e Figueiredo, D. (2003). Qualidade de vida e bem-estar dos idosos: um estudo exploratório na população portuguesa, Revista Saúde Pública, 37(3), 364-371. 
Stutzman, F. (2006). Student life on the facebook. Acedido em 20/02/2011. Disponível em: <http://ibiblio.org/fred/facebook/stutzman_fbook.pdf>.

Taborda, M. (2010). A utilização da internet em Portugal 2010. LINI - Lisbon Internet and Networks International Research Programme. Lisboa.

Tapscott, D. \& Williams, A. (2007). Wikinomics, a nova economia de multidões inteligentes. Editora Quidnovi.

Tredinnick, L. (2006). Web 2.0 and business: A pointer to the intranets of the future, Business Information Review, 23(4), 228-234.

Vasconcelos, V. \& Campos, P. (2010). Distributed informal information systems for innovation: An empirical study of the role of social networks, in Varajão, J. et al. (Eds.), Enterprise Information Systems. Part II CCIS 110, Springer-Verlag, 208217.

Weber, L. (2009). Marketing to the social web: How digital customer communities build your business. New Jersey: John Wiley \& Sons.

Wilson, J. (2009). Social networking: The business case, Engineering and Technology, 4(10), 54-56.

Yap, R \& Robben, J. (2010). A model for leveraging social learning technologies in corporate environments. Proceeding of the 7 th International Conference on Networked Learning.

Zhou, L., Wu, W. \& Luo, X. (2007). Internationalization and the performance of bornglobal SMEs: The mediating role of social networks, Journal of International Business Studies, 38, 673-690. 